\title{
Tendencia de los niveles de plomo en la atmósfera de la zona metropolitana de la Ciudad de México. 1988-1998
}

\author{
Marlene Cortez-Lugo, Ing, ${ }^{(1,2)}$ Martha Ma Téllez-Rojo, Mat, M en C, D r Sc, ${ }^{(1)}$ \\ Héctor Gómez-Dantés, MC, M en $C,{ }^{(3)}$ Mauricio Hernández-Avila, MC, Dr Sc. ${ }^{(1)}$
}

\begin{abstract}
Cortez-Lugo M,Téllez-Rojo MM, Gómez-Dantés H, Hernández-Avila $M$. Tendencia de los niveles de plomo en la atmósfera de la zona metropolitana de la Ciudad de México. 1988-1998. Salud Publica Mex 2003;45 supl 2:S196-S202. El texto completo en inglés de este artículo está disponible en: http://www.insp.mx/salud/index.html
\end{abstract}

\section{Resumen}

Objetivo. Describir las tendencias del plomo atmosférico asociadas a la eliminación paulatina del plomo de la gasolina y el uso de convertidores catalíticos en el auto transporte de la Ciudad de México, de 1988 a 1998. Material y métodos Estudio de series de tiempo que incluye las mediciones atmo sféricas de plomo que se obtuvieron, entre 1988 y 1998, de nueve estaciones de la red manual de monitoreo atmo sférico de la Ciudad de México. Las inter venciones evaluadas fueron la introducción de gasolina Magnasin, el uso de convertidores catalíticos y el descenso en la concentración de plomo hasta su eliminación total de la gasolina utilizada en los auto transportes. Se describen las tendencias de plomo por monitor, por zona geográfica, utilizando un modelo de regresión que incorpora una estructura de autocorrelación. Resultados La introducción de gasolina sin plomo y de los convertidores catalíticos se asoció con un descenso en la concentración de plomo atmosférico en 23\%, la reducción paulatina del plomo en la gasolina ocasionó una reducción acumulada de $89 \%$ en todas las zonas estudiadas. Conclusiones. La introducción de la gasolina sin plomo ha logrado abatir las concentraciones ambientales de este contaminante de una manera importante. El texto completo en inglés de este artículo está disponible en:http:/ /www.insp.mx/salud/index.html

Palabras clave: plomo; contaminación ambiental; gasolina; Ciudad de México

\section{Cortez-Lugo M,Téllez-Rojo MM,}

Gómez-Dantés H, Hernández-Avila M.

Trends in atmospheric concentrations of lead

in the Metropolitan Area of Mexico City. 1988-1998.

Salud Publica Mex 2003;45 suppl 2:S196-S202.

The English version of this paper

is available at: http://www.insp.mx/salud/index.html

\begin{abstract}
A bstract
Objective. To describe the trends in airborne lead levels and their association with the control measures implemented from 1988 to 1998 to phase out lead from gasoline that included the introduction of a lead-free gasoline and the use of catalytic converters in automobiles in Mexico City. Material and Methods Data of atmospheric lead levels were obtained from the 9 field stations included in the local air quality monitoring network (RMMA). Trends for airborne lead are shown by monitor, monitoring area and for the entire metropolitan zone. Analysis of data was performed using linear regression models including autocorrelation analysis. The models were validated by standard diagnostic techniques using student residuals analysis. $\mathbf{R e}$ sults. The introduction of lead-free gasoline and the use of catalytic converters decreased the airborne lead levels by $23 \%$ while the slow elimination of lead from gasoline produced a cumulative decrease of $89 \%$ during the period and in all areas studied. Conclusions The strategy implemented to reduce sources of airborne lead pollution had an important impact on atmospheric lead levels. Health risks of this environmental hazard are now primarily due to less important sources and to particular occupational exposures. The English version of this paper is available at: http:// www.insp.mx/salud/index.html
\end{abstract}

Key words:lead; environmental health; gasoline; Mexico City

(1) Centro de Investigación en Salud Poblacional, Instituto N acional de Salud Pública. Cuernavaca, Morelos, México.

(2) American British Cowdray, Medical Center. México, D F, México.

(3) División Técnica de Información Estadística en Salud de la Dirección de Prestaciones Médicas, Instituto Mexicano del Seguro Social. México, DF, México.

Fecha de recibido: 12 de mayo de 2003 • Fecha de aprobado: 17 de septiembre de 2003 Solicitud de sobretiros: D r. Mauricio Hernández Avila. Instituto N acional de Salud Pública. Avenida Universidad 655 colonia Santa María A huacatitlán 62508. Cuernavaca, Morelos, México. Correo electrónico:mhernan@ correo.insp.mx 
E 1 acelerado crecimiento de la zona metropolitana de la Ciudad de México (ZMCM) durante las últimas dos décadas la ha transformado en una de las megaurbes más importantes en el mundo. En la ZMCM habita $20 \%$ de la población total del país, ${ }^{1}$ se centraliza parte importante de la actividad industrial, política y comercial, ${ }^{2}$ pero también se concentran riesgos diversos entre los que destacan el incremento en los niveles de contaminación ambiental que responden a los ritmos y efectos indirectos del consumo de combustibles. ${ }^{3}$ La migración del campo a la ciudad, el crecimiento en la densidad poblacional, el proceso de industrialización, las condiciones geográficas del Valle de México, la desorganizada red de transporte público, la demanda en el uso de automóviles, la insuficiencia de la red vial, junto con la producción y el consumo de gasolina, surgen como las determinantes más importantes del deterioro en las condiciones y la calidad de la atmósfera en la ZMCM.

Si bien existen diferentes fuentes emisoras de contaminantes atmosféricos, el plomo ambiental se reconoce como uno de los riesgos más importantes en la salud debido a sus efectos deletéreos, acumulativos y permanentes sobre el desarrollo neurológico de los niños. ${ }^{4,5}$ Los efectos nocivos del plomo se amplifican en la medida en que aumenta su concentración en sangre, y el amplio espectro de daños que produce incluyen la disminución en la producción de hemoglobina y la anemia, afecta el metabolismo de la vitamina D, disminuye la velocidad de conducción nerviosa, produce daño renal, hipertensión arterial, y encefalopatía, daña el desarrollo cognoscitivo y físico de los niños, y puede producir hasta la muerte. ${ }^{6}$

Debido a sus propiedades físicas y a que es un metal dúctil, maleable y resistente a la corrosión, el plomo ha sido utilizado en muy diversas aplicaciones dentro de las industrias metalúrgica y eléctrica para la producción de materiales, objetos y utensilios de uso cotidiano. Los pigmentos con plomo son muy apreciados por su colorido y bajo costo. En la industria del transporte se usa como proveedor de octanaje para la gasolina y lubricador de válvulas. Las fuentes de exposición al plomo en las áreas urbanas -ocupacional y poblacional- se asocian con los desechos y emisiones de industrias tan diversas como la metálica, la cerámica, la producción de latas para envasar alimentos, pinturas, pigmentos, juguetes y baterías, mientras que la exposición doméstica se realiza a través del contacto con las tuberías de plomo y del uso de la cerámica vidriada para cocinar y consumir alimentos.

México se encuentra en el decimotercer lugar entre los países productores de plomo, con una producción cercana a las 140000 toneladas en 1990, de las cuales se exportó 60\%. ${ }^{7}$ También se estima que alrededor de $30 \%$ del total de las emisiones de plomo en la atmósfera en el país son generadas en la ZMCM y que, en promedio, durante la década de los ochenta se liberaron alrededor de 1500 toneladas cúbicas de plomo en la atmósfera de la Ciudad de México asociadas sólo con la combustión de gasolina. ${ }^{8}$

Debido a que el uso de gasolina con plomo produce emisiones de este contaminante hacia el ambiente y a que los daños asociados con el plomo producen una carga importante de morbilidad y discapacidad, sobre todo en la infancia, el Banco Mundial lanzó la iniciativa de eliminarlo de las gasolinas en el ámbito mundial. En vista de que esta iniciativa afectaba de manera importante el diseño y producción de automotores e imponía nuevos procesos productivos para la industria petrolera y de gasolinas, este proceso se fue instrumentando de manera paulatina en diversos países. En México inició en 1986 con reducciones en el contenido de plomo en las gasolinas y, en septiembre de 1990, con la introducción de la gasolina Magnasin y la incorporación de los convertidores catalíticos en los automóviles de nueva manufactura. A partir de entonces se fueron implantando nuevas reducciones en las concentraciones de plomo en la gasolina, hasta lograr su total eliminación en 1997.

El objetivo de este trabajo es describir la tendencia en la concentración de plomo atmosférico, de 1988 a 1998 en la ZMCM, vinculada a los cambios introducidos en la formulación de las gasolinas para autotransportes, el uso de convertidores catalíticos y, de manera indirecta, por la reducción de su uso en otras actividades industriales.

\section{Material y métodos}

Este estudio de series de tiempo analiza la tendencia de las concentraciones del plomo en la atmósfera de la ZMCM durante el periodo de 1988 a 1998. La información sobre las concentraciones de plomo se obtuvo de las mediciones de partículas suspendidas totales (PST) realizadas por la red manual de monitoreo ambiental (RMMA) a través de las 20 estaciones distribuidas en diversas áreas de la ZMCM. La RMMA realiza determinaciones de plomo desde 1986, pero en sus inicios estas mediciones se efectuaron de manera irregular y no se implantaron simultáneamente en todas las estaciones. Fue hasta 1988 cuando el monitoreo se hizo de manera continua y a partir de entonces existen registros más completos para la mayoría de las estaciones.

El criterio para seleccionar las estaciones fue que contaran con mediciones regulares de plomo durante al menos nueve de los 11 años del periodo en estudio 
(1988-1998). De las 20 estaciones de la RMMA, sólo nueve contaron con mediciones continuas de plomo que fueran suficientes para ser incluidas en el análisis estadístico. La distribución geográfica de las nueve estaciones seleccionadas fue la siguiente: una pertenece a la zona noroeste (Tlalnepantla), dos a la zona noreste (Xalostoc y Cerro del Tepeyac), dos a la zona centro (Merced y Museo de la Ciudad de México), tres a la zona suroeste (Comisión Federal de Electricidad, Felipe Angeles y Pedregal) y una a la zona sureste (Cerro de la Estrella).

El plomo atmosférico en la ZMCM se mide, a través de su extracción del material particulado, con soluciones de ácido nítrico y clorhídrico y se analiza por medio de espectrofotometría de absorción atómica. Las mediciones de plomo se hacen cada seis días, en colecciones de material particulado de 24 horas de duración y se realizaron agregados mensuales para derivar concentraciones mensuales promedio.

La Norma Oficial Mexicana de Plomo (NOM-026SSA1-1993), publicada en el Diario Oficial de la Federación el 23 de diciembre de 1994, establece que el límite de plomo permisible en la atmósfera no debe exceder un promedio trimestral de $1.5 \mu \mathrm{g} / \mathrm{m}^{3}$, y éste es considerado un umbral de seguridad que no afecta la salud de los habitantes de la ZMCM.

Las medidas puestas en práctica para reducir el contenido de plomo en la gasolina distribuida en el ZMCM empezaron en 1986 con la clausura de la refinería de Atzcapotzalco y la disminución del tetraetilo de plomo en la gasolina Extra; en 1987 inicia el Programa Hoy No Circula en forma voluntaria y no es sino hasta 1989 cuando se pone en práctica en forma obligatoria. A partir de septiembre de 1990 se realizan intervenciones específicas que introducen la gasolina Magnasin y los convertidores catalíticos en los automotores de manufactura nueva (lo que en nuestro estudio llamaremos "medida 1"). En septiembre de 1991 se estableció la especificación de que la gasolina contuviera sólo de 0.5 a $1.0 \mathrm{ml}$ de tetraetilo de plomo (TEP) por galón (medida 2), reemplazando el límite anterior de 3 a $5 \mathrm{ml} \mathrm{TEP/gal.} \mathrm{En} \mathrm{1992,} \mathrm{este} \mathrm{rango} \mathrm{se} \mathrm{redujo} \mathrm{a} 0.2$ a $0.3 \mathrm{ml} \mathrm{TEP} /$ gal (medida 3); en 1994 se restringió a un rango de 0.1 a $0.2 \mathrm{ml} \mathrm{TEP/gal} \mathrm{(medida} \mathrm{4)} \mathrm{hasta} \mathrm{que,}$ finalmente, en agosto de 1997 se eliminó totalmente el uso del plomo en la gasolina (medida 5).

Las mediciones de plomo en aire se tomaron como la variable dependiente. Las variables independientes consideradas fueron tanto el tiempo como las medidas instrumentadas; estas últimas se incorporaron como variables indicadoras con valor 0 durante el tiempo previo a ser instrumentadas y con valor 1 a partir del inicio de su vigencia.
Para el análisis de los datos se utilizó el paquete de STATA 5.0. Se realizó un análisis descriptivo de la información en tres niveles de agregación: por monitor, por zona de la ZMCM -promediando las mediciones de los monitores correspondientes a cada zona- y, finalmente, se realizó un análisis global que integró la información de todos los monitores. Puesto que el plomo en la atmósfera no mostró un comportamiento probabilístico gaussiano tuvo que ser transformado logarítmicamente para normalizar la variable. Inicialmente se generó un modelo de regresión lineal estándar. Debido a la naturaleza seriada y la dependencia de las mediciones se utilizó el estadístico de Durbin-Watson (DW) para evaluar una autocorrelación de primer orden. Para evaluar las tendencias en los niveles de plomo ambiental en el tiempo se utilizó una generalización de la regresión lineal estándar -Regresión de Cochrane-Orcutt-,${ }^{9}$ que propone la estimación de los parámetros de regresión y sus errores estándar a través de un proceso iterativo que incorpora una estructura de autocorrelación sin la restricción de ser de primer orden, como en el caso de la DW, y que además permite la interpretación usual de los coeficientes. Los modelos propuestos se validaron mediante técnicas de diagnóstico estándar, utilizando los residuos estudentizados.

Para poder visualizar la tendencia que describió estos datos a través del tiempo, se ajustó la curva utilizando la herramienta gráfica basada en la técnica no paramétrica de Lowess. ${ }^{10,11}$ En esencia, lo que se logra al promediar los valores de cada medición en el tiempo es el suavizamiento de una curva para visualizar la tendencia de los niveles de plomo.

\section{Resultados}

En el cuadro I se presentan las estadísticas descriptivas de las nueve estaciones de monitoreo consideradas en el análisis donde se enfatiza el número de días con medición, por haberse considerado un criterio de inclusión en el mismo. La zona con concentraciones mayores de plomo fue la noroeste, representada por la estación Xalostoc $\left(1.22 \mu \mathrm{g} / \mathrm{m}^{3}\right)$ y Tepeyac $(0.79$ $\left.\mu \mathrm{g} / \mathrm{m}^{3}\right)$, mientras que la zona sureste fue aquella donde se presentaron los niveles más bajos (Pedregal 0.36 $\left.\mu \mathrm{g} / \mathrm{m}^{3}\right)$.

El comportamiento del plomo atmosférico en la ZMCM, para cada una de las estaciones de monitoreo en el primero y el último año del periodo de estudio, muestra el descenso de este contaminante en todas las estaciones. En todas éstas se rebasó el límite de plomo permisible en la atmósfera de $1.5 \mu \mathrm{g} / \mathrm{m}^{3}$ durante los primeros años, pero descendió en la última etapa 


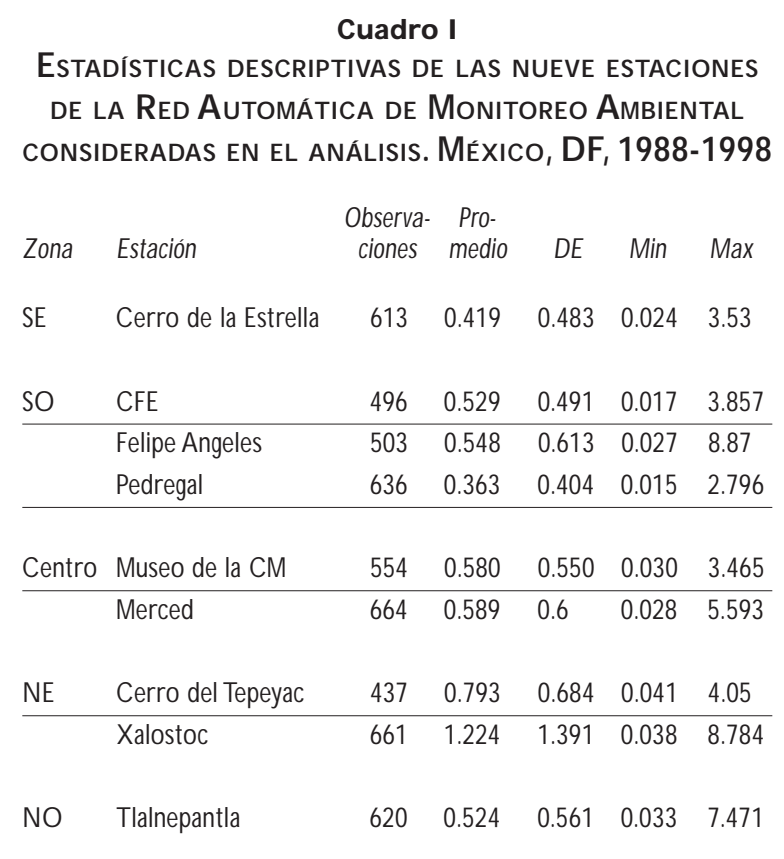

del periodo estudiado, además, a partir de 1993 ya no se presentaron excedencias.

Las tendencias del plomo por zona muestran un comportamiento decreciente significativo, y la magnitud del descenso anual es muy similar en todas las zonas del Distrito Federal (DF) siendo ésta más marcada en las zonas del sur de la ZMCM (cuadro II).

Para medir el efecto que tuvieron las medidas implantadas sobre los niveles de plomo ambiental, el análisis se realizó incorporando las medidas 1 a 5 como variables indicadoras a partir de la fecha de su vigencia (cuadro II). La introducción de la gasolina sin plomo y de convertidores catalíticos en los automóviles nuevos en septiembre de 1990 tuvo un efecto significativo sobre los niveles registrados de plomo en el aire, logrando una disminución promedio estimada de $22.9 \%$. Asimismo, la reducción de la especificación de plomo en la gasolina de 0.5-1.0 ml TEP/gal a 0.3-0.5 $\mathrm{ml} \mathrm{TEP/gal} \mathrm{en} \mathrm{septiembre} \mathrm{de} \mathrm{1991,} \mathrm{tuvo} \mathrm{también} \mathrm{un}$ impacto significativo. Para ese momento, cuando las dos medidas ya estaban vigentes, se estimó que el logaritmo del plomo tuvo una reducción de 0.73 atribuible a ellas, lo cual se traduce en un decremento medio estimado en los niveles de plomo de $52 \%$. El efecto de la nueva reducción de la especificación de plomo a 0.2-0.3 $\mathrm{ml} \mathrm{TEP} /$ gal de noviembre de 1992 promovió un decremento de 0.58 sobre el plomo transformado logarítmicamente, ocasionando una reducción acumulada estimada de $73 \%$ en el plomo ambiental. La nueva reducción a 0.1-0.2 $\mathrm{ml}$ TEP/gal puesta en mar-

\begin{tabular}{|c|c|c|c|c|}
\hline \multicolumn{5}{|c|}{ 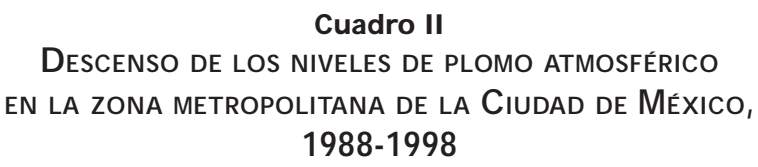 } \\
\hline Zona/estación & $\begin{array}{l}\text { bservaciones } \\
\text { promedio }\end{array}$ & $\begin{array}{l}\% \text { de descenso } \\
\text { anual }\end{array}$ & & $\begin{array}{c}R^{2} \\
\text { ajustada }\end{array}$ \\
\hline \multicolumn{5}{|l|}{ Sureste } \\
\hline Cerro de la Estrella & 533 & $5.6 \%$ & $5.2-6.0 \%$ & 0.58 \\
\hline \multicolumn{5}{|l|}{ Suroeste } \\
\hline CFE, Pedregal y Felipe Angeles & es 589 & $5.7 \%$ & $5.3-6.1 \%$ & 0.59 \\
\hline \multicolumn{5}{|l|}{ Centro } \\
\hline Merced y Museo de la CM & 599 & $4.8 \%$ & $4.5-5.1 \%$ & 0.62 \\
\hline \multicolumn{5}{|l|}{ Noreste } \\
\hline X alostoc y Cerro de Tepeyac & 585 & $4.9 \%$ & $4.5-5.2 \%$ & 0.54 \\
\hline \multicolumn{5}{|l|}{ Noroeste } \\
\hline Tlalnepantla & 538 & $4.2 \%$ & $3.9-4.6 \%$ & 0.53 \\
\hline Global & 608 & $4.8 \%$ & $4.5-5.1 \%$ & 0.58 \\
\hline
\end{tabular}

cha en noviembre de 1994, condujo a una reducción acumulada de $82 \%$ y, finalmente, la eliminación total de plomo en los diferentes tipos de gasolina, en agosto de 1997, logró una disminución acumulada de 89\%. Estos resultados se muestran en el cuadro III y gráficamente en la figura 1.

Como se mencionó, se decidió excluir 11 monitores del análisis por el excesivo número de datos faltantes que presentaron durante el periodo de estudio. El coeficiente de correlación entre la media de los monitores incluidos y los no incluidos fue de 0.84 , siendo altamente significativo (valor $p<0.01$ ). Asimismo, el coeficiente de correlación por zona de la ciudad entre los monitores incluidos y no incluidos fue de $0.86,0.69$, $0.67,0.62$ y 0.34 para las zonas sureste, suroeste, noreste, noroeste y centro, respectivamente. Por lo anterior, no consideramos que esta selección pudiera tener un efecto diferente al reportado con todos los monitores y al impacto sustancial de los resultados aquí presentados.

\section{Discusión}

Las concentraciones de plomo en la atmósfera de la ZMCM han descendido de manera progresiva durante los últimos 10 años. Este comportamiento podría haber sido el resultado de las diversas acciones tendientes a disminuir el uso de plomo en diversos productos, 


\section{Cuadro III \\ IMPACTO DE LAS MEDIDAS IMPLANTADAS EN LA REDUCCIÓN DEL PLOMO ATMOSFÉRICO en la zona metropolitana de la Ciudad de México, 1988-1998}

Medidas implantadas
Reducción estimada en la media
geométrica

Reducción acumulada en la media geométrica

Reducción acumulada atribuible a las diversas medidas (\%)

1) Introducción de gasolina Magnasin y convertidores catalíticos (a partir de septiembre de 1990)

2) Cambio en dosificación a de 0.5 a $1.0 \mathrm{ml}$.TEP/gal (a partir de 1991)

$\begin{array}{ll} & -0.26 \\ \text { 1991) } & -0.47 \\ 92) & -0.58 \\ 94) & -0.39\end{array}$

$\begin{array}{lll}-0.26 & -0.26 & 22.9 \\ -0.47 & -0.73 & 52 \\ -0.58 & -1.31 & 73 \\ -0.39 & -1.70 & 82 \\ -0.51 & -2.21 & 89\end{array}$

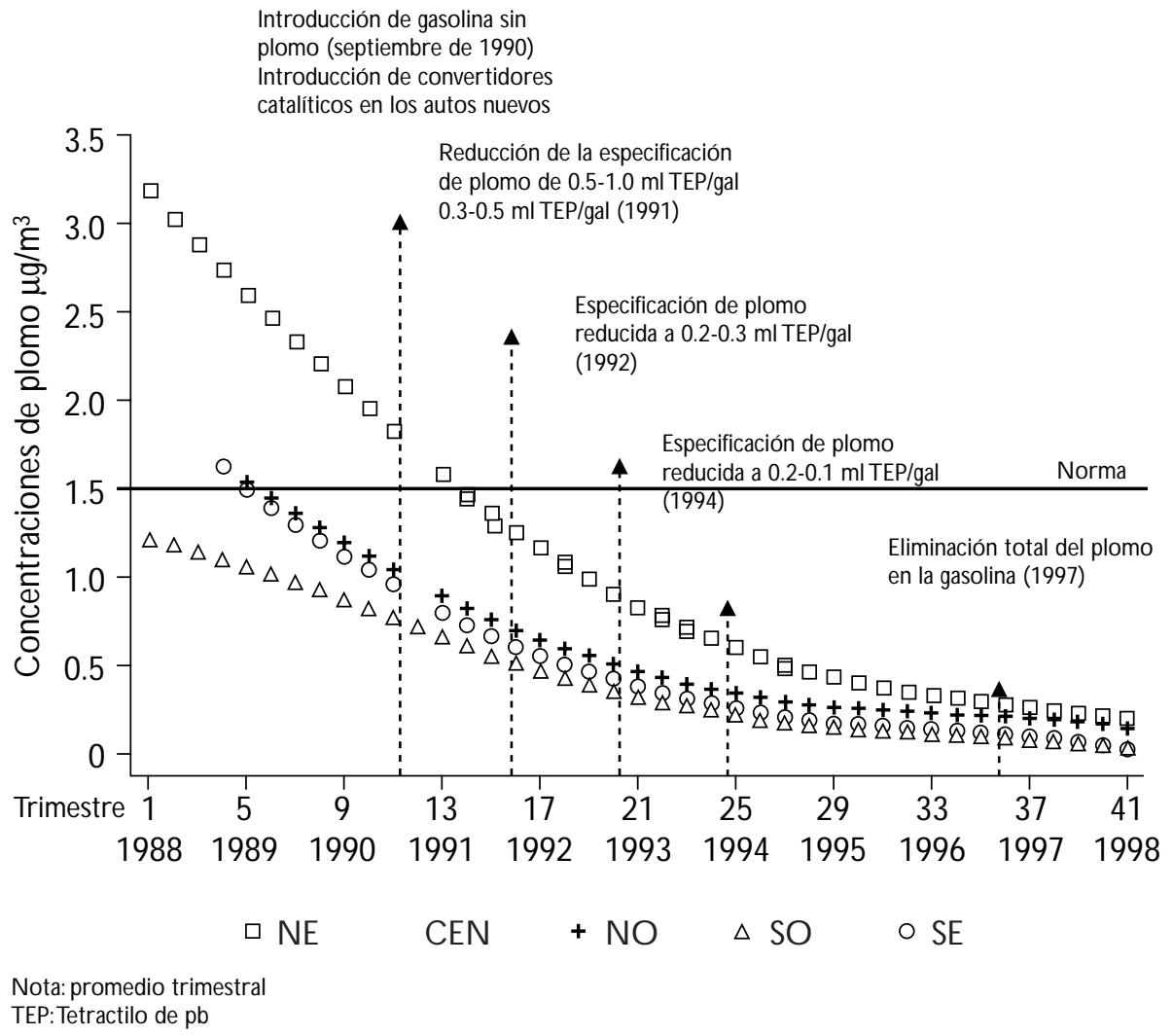

Figura 1.Tendencia de los niveles de plomo atmosférico en la zona metropolitana de la Ciudad de México, 1988-1998

principalmente la gasolina, a la eliminación de la refinería de Azcapotzalco, así como a la reducción en la circulación de los automóviles con el Programa Hoy No Circula, puesto en marcha en forma obligatoria desde 1989. Otras medidas para reducir la exposición al plomo contemplaron el control de las concentraciones del metal en agua, en la descarga de aguas residuales, en los residuos sólidos, alimentos, bebidas, medicamentos y cosméticos. En 1991 se estableció un convenio con las industrias fabricantes de envases me- 
tálicos, pinturas y pigmentos, y con los artesanos, para promover a corto, mediano y largo plazo la reducción y eliminación de plomo en los productos de consumo. La producción de latas con soldadura de estaño y plomo se eliminó desde 1992 y la importación de productos enlatados tiene una vigencia estricta desde mayo de $1993 .{ }^{12}$

Si bien la concentración de plomo atmosférico ya marcaba una tendencia hacia el descenso antes de que se instrumentaran las medidas evaluadas, la tendencia se intensificó gracias a la eliminación del plomo en la gasolina, ya que su contribución como fuente emisora es mucho más importante que las generadas por otras actividades industriales. Las primeras medidas implantadas en este sentido se iniciaron con una reducción en el tetraetilo de plomo en las gasolinas, que podría explicar la tendencia decreciente previa a la introducción de las primeras gasolinas sin plomo. Todas ellas han tenido un impacto significativo en los niveles de plomo en aire y su impacto en la salud se ha documentado en estudios que monitorearon los niveles de plomo en sangre en diversos grupos de población antes, durante y después de instrumentadas las medidas.

El descenso en los niveles de plomo en aire desde 1988, cuando se registraron niveles promedio de $1.95 \mu \mathrm{g} / \mathrm{m}^{3}$ que bajaron a $0.28 \mu \mathrm{g} / \mathrm{m}^{3}$ en $1994,{ }^{13}$ se ha asociado con el descenso en los niveles de plomo en sangre en diversos estudios en la población general. ${ }^{14-16}$ En 1980, por ejemplo, se reportaron niveles medios de plomo en sangre de $24 \mu \mathrm{g} / \mathrm{dl}$ en una cohor- te de 85 profesores. ${ }^{17}$ Niveles elevados de plomo en sangre también se encontraron en un estudio en pacientes hospitalizados de la Ciudad de México (19911992). Los monitoreos periódicos realizados en la ZMCM desde 1991 reportaron un descenso constante en los niveles de plomo en sangre en la población en general, aunque se estima que entre 10 a $20 \%$ de los niños en la Ciudad de México aún tienen niveles de plomo que exceden los $10 \mu \mathrm{g} / \mathrm{dl}^{18-20}$

La experiencia de la eliminación del plomo en la gasolina y su impacto en la salud de la población reportada para la ZMCM se une a la larga serie de intervenciones similares que se han desarrollado en diferentes países en las últimas dos décadas (cuadro IV). De estos estudios se desprende lo notable de la respuesta internacional a un problema de salud pública de importancia global. Si bien la mayoría de los estudios y las intervenciones fueron realizados en países desarrollados, esto no es indicativo de que los países en desarrollo no puedan -debido a los costos y las reformas tecnológicas necesarias-incorporarse a esta estrategia preventiva. Las poblaciones estudiadas también demuestran el interés en los grupos más vulnerables a la exposición a plomo. Predominan los estudios sobre niños y escolares ${ }^{21-25}$ y en mujeres embarazadas. ${ }^{26,27}$ Asimismo, se incluyen reportes de los niveles de plomo en sangre en la población en general, ${ }^{28,29}$ en la población adulta, ${ }^{30,31}$ y en grupos específicos como atletas ${ }^{32}$ y donadores de sangre. ${ }^{33}$ Las reducciones en las concentraciones de plomo en las diferentes gasolinas, sin llegar a la eliminación total,

\section{Cuadro IV \\ Resumen de estudios realizados después de la Reducción de plomo en las gasolinas}

\begin{tabular}{|c|c|c|c|c|}
\hline Autores & País & Años comparados & Diseño de estudio & $\begin{array}{l}\text { Cambio de la concentración } \\
\text { de plomo en la gasolina/el aire }\end{array}$ \\
\hline Rabinowitz and N eedleman (1983) & EUA & 1979-1981 & Transversal & $250 * 10^{6}$ gal por mes a $75^{*} 10^{6}$ por mes \\
\hline Maravelias et al (1998) & Grecia & 1982-1996 & Transversal & 3.21 a $0.4 \mu \mathrm{g} / \mathrm{m}^{3}$ \\
\hline H ayes et al (1994) & EUA & 1968-1988 & Transversal & 0.9 a $0.1 \mu \mathrm{g} / \mathrm{m}^{3}$ \\
\hline Groble (1992) & Sudáfrica & 1984-1990 & Transversal & $0.8 \mathrm{~g} / \mathrm{l} \mathrm{a} 0.4 \mathrm{~g} / \mathrm{l}$ \\
\hline Q uinn and D elves (1989) & Inglaterra & 1985-1986 & Longitudinal y transversal & 0.4 a $0.15 \mathrm{~g} / \mathrm{l}$ \\
\hline Bono et al (1995) & Italia & 1985-1994 & Transversal & 0.4 a $0.15 \mathrm{~g} / \mathrm{l}$ \\
\hline W ang et al (1997) & Canadá & 1982-1990 & Transversal & $2.6 * 10^{9}$ a $1.9 * 10^{7} \mathrm{~g}$ de pb por año \\
\hline W eitlisbach et al (1995) & Suiza & 1984-1993 & Transversal & 0.40 a $0.15 \mathrm{~g} / \mathrm{l}$ \\
\hline Hinton et al (1986) & N ueva Zelanda & 1978-1985 & Transversal & Sin cambio \\
\hline Taylor et al (1995) & Australia & 1993-1979 & Transversal & $3.3 \mu \mathrm{g} / \mathrm{m}^{3}$ a $0.96 \mu \mathrm{g} / \mathrm{m}^{3}$ \\
\hline Schuhmacher et al (1996) & Tarragona, España & 1990-1995 & Transversal & 0.40 a $0.15 \mathrm{~g} / \mathrm{l}$ \\
\hline Maresky and Grobles (1993) & Capetown, Sudáfrica & 19841990 & Transversal & $0.83 \mathrm{~g} / /$ a $0.4 \mathrm{~g} / \mathrm{l}$ \\
\hline
\end{tabular}

Fuente: Modificado de 0 ptions for Monitoring Biologica and Environmental Lead During the Phase-out of Lead in Gasoline in Latin America \& The Caribbean in Environmental Health Project. Activity Report N 0. 47, 1999 
son muy similares a las alcanzadas en México. La media en niveles de plomo en sangre descendió casi 50\% en la mayoría de los estudios.

Los resultados que aquí se reportan resumen la esencia y la importancia de las intervenciones en salud pública. Conocido el daño y el peso atribuibles a la exposición a un contaminante específico, la puesta en marcha de acciones dirigidas a reducir dicha exposición en la población se manifiesta en un control efectivo de la emisión del metal en la atmósfera y se acompaña de una reducción importante en los niveles de plomo en sangre de la población que habita en la ZMCM. La oportunidad con la que se realizaron las medidas, lo complejo de su puesta en práctica y el alcance de su impacto merece un especial reconocimiento. En el éxito alcanzado confluyen un número importante de actores que laboran en la industria, en el sector salud, en el gobierno, en las instituciones académicas y de investigación y en la sociedad civil. Un rasgo también destacable de lo que aquí se reporta es la naturaleza permanente del impacto, ya que las medidas adoptadas eliminaron el riesgo de una fuente de emisión cuya capacidad de generación era excesiva, continua y que tendería hacia el incremento, de no haberse realizado.

\section{Referencias}

1. N egrete-Salas M. D inámica demográfica en la Ciudad de México: la Ciudad de México en el fin del segundo milenio. México, DF: El Colegio de México, 2000:247-255.

2. Sobrino J. Inversión pública federal en la Ciudad de México: la Ciudad de México en el fin del segundo milenio. México, DF: El Colegio de México, 2000:220-226.

3. Lezama JL. Contaminación del aire en la Ciudad de México: la Ciudad de México en el fin del segundo milenio. El Colegio de México, 2000:461-468

4. N eedleman $H$, Bellinger $D$, Leviton A. D eficit in psychologic and classroom performance of children with elevated dentine levels. N Engl J Med 1979;300:689.

5. N eedleman H, Gatsonis C. Low-level lead exposure and the IQ of children. J Am Med Assoc 1990;263:673-678.

6. Howson CP, Hernández-Avila M, Rall DP, Ed. Lead in the Americas:A call for action. Toluca, México: Institute of Medicine of USA/N ational Institute of Public Health, 1996.

7. Secretaría de Energía, Minas e Industria Paraestatal. Dirección General de Minas: informes mensuales. México DF, SEMIP, 1989. 8. C ontreras R. The case of Mexico. En: Finkelman J, C orey G, Calderón $R, E d$. Environmental epidemiology: A project in Latin America and The Caribbean. Metepec, México: Panamerican Health 0 rganization 1993:114-118.

9. Johnston J. Econometric methods. $3^{d}$ Ed. N ueva York (N Y): McG raw-Hill, 1984

10. Fan J, G ybels I. Local polynomial modelling and it's applications. Great Britain: C hapman \& Hall, 1996.

11. Hamilton LC. Regression with Graphics. A second course in applied statistics. Brooks/C ole Publishing Company, 1992.
12. Jiménez-C orona A, Gómez-D antés H, Tapia CR, 0 laiz F. N iveles de plomo en sangre de cordón umbilical en hospitales de la Ciudad de México (1992-1994). Gac Med Mex 1996;132(4):447-450.

13. Romieu I, Palazuelos E, Meneses $F$ et al.Vehicular traffic as a determinant of blood-lead levels in children: A pilot study in Mexico City. Arch Environ Health 1992;47(4):246-249.

14. D riscoll W, Mushak P, G arfias J. Reducing lead in gasoline: Mexico's experience. Environ Sci Technol 1992;26(9)1702-1705.

15. Romieu I, Palazuelos E, Hernández-Avila M et al. Sources of lead exposure in Mexico City. Environ Health Perspect 1994;102(4):384-389. 16. Rothenberg S, Schnaas L, Perroni E, Hernández RM, Karchmer S. Secular trend in blood lead levels in a cohort of Mexico City children. Arch Environ Health 1998:53:231-235.

17. Alliance to end childhood lead poisoning and environmental defense fund. The global dimensions of lead poisoning, W ashington, DC;AECLP/ ED F, 1994:52-54.

18. Jiménez C, Romieu I, Palazuelos E, Muñoz I, Cortez M, Rivero A et al. Factores de exposición ambiental y concentraciones de plomo en sangre en niños de la Ciudad de México. Salud Publica Mex 1993;35:599-606.

19. Romieu I. Lacasaña M. Prevalence of exposure and data quality of lead contamination in Latin America and The Caribbean. En: Houson CP, Hernández-Avila M, Rall DP, Ed. Lead in the A mericas:A call for action.

Toluca, México: Institute of Medicine of USA/N ational Institute of Public Health, 1996:77-83.

20. 0 laiz G, Fortoul T, Rojas R. Risk factors for high levels of lead in blood of schoolchildren in Mexico City. Arch Environ Health 1996;51(2): 122-126.

21. Maravelias C, A thansalelis S, D ona S, C hatzioanov A, Priftis C, Koutselinis $A$ et al. Reduction of lead pollution in G reece during the past two decades. Arch Environ Health 1998;53:424-426.

22.W ang ST, Pizzolato S, D emshar HP, Smith LF. D ecline in blood lead in 0 ntario children correlated to decreasing consumption of leaded gasoline, 1983-1992. Clin Chem 1997;43:1251-1252.

23. Rothenberg SJ, Schnaas L, Perroni E, Hernández RM, Karchmer S. Secular trend in blood lead levels in a cohort of Mexico City children. Arch Environ Health 1998:53:231-235.

24.Taylor R, Bazelmans J, Golec R, 0 akes S. D eclining blood lead levels in Victorian children. Aust J Public Health 1995;19:455-459.

25. Hayes E, Mc Elvaine M, $O$ rbach H, Fernández AM, Lyne S, Matte TD. Long-term trends in blood lead levels among children in Chicago: Relationship to air lead levels. Pediatrics 1994;93(2):195-200.

26. Rabinowitz M, N eedleman H. Petrol lead sales and umbilical cord blood lead levels in Boston, Massachusetts. Lancet 1983;1(8314-5):63. 27. Hinton D, Coope P, Malpres W, Janus ED. Trends in blood lead levels in Christchurch, (NZ) and environs 1978-85. J Epidemiol Commun Health 1986;40:244-248.

28. W eitlisbach V, Rickenbach M, Berode M, G uillemin M.T ime trend and determinants of blood lead levels in a Swiss population over a transition period (1984-1993) from leaded to unleaded gasoline use. Environ Res 1995;68:82-90.

29. Q uinn M, D elves H. UK blood lead monitoring programme 1984-87: Results for 1986. Human Toxicol 1989;6:205-220.

30. Schuhmacher M, Belles M, Rico A, D omingo JL, C orbella J. Impact of reduction of lead in gasoline on the blood and hair lead levels in the population of Tarragona Province, Spain, 1990-95. Sci Total Environ 1996;184(3):203-209.

31. Maresky L, G robler S. Effect of the reduction of petrol lead on the blood lead levels of South Africans. Sci Total Environ 1993;136(1-2):43-48. 32. G robler S, Maresky L, Kotze T. Lead reduction of petrol and blood lead concentrations of athletes. Arch Environ Health 1992;47:139-142. 33. Bono R, Pignata C, Scursatone E, Rovene R, N atale P, G illi G. Updating about reductions of air and blood lead concentrations in Turin, Italy, following reductions in the lead content of gasoline. Environ Res 1995;70:30-34. 